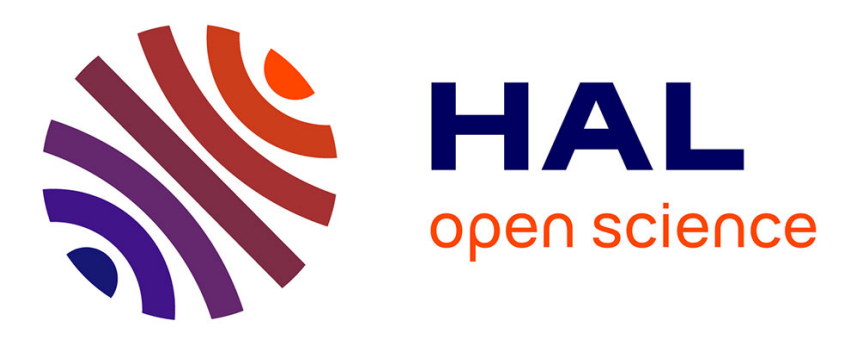

\title{
Stressed silicon nitride nanomechanical resonators at helium temperatures
}

\author{
Martial Defoort, Kunal Lulla, Christophe Blanc, Hossein Ftouni, Olivier \\ Bourgeois, Eddy Collin
}

\section{- To cite this version:}

Martial Defoort, Kunal Lulla, Christophe Blanc, Hossein Ftouni, Olivier Bourgeois, et al.. Stressed silicon nitride nanomechanical resonators at helium temperatures. Journal of Low Temperature Physics, 2013, 171 (5-6), pp.731-736. 10.1007/s10909-012-0693-5 . hal-00861790

\section{HAL Id: hal-00861790 \\ https://hal.science/hal-00861790}

Submitted on 17 Dec 2013

HAL is a multi-disciplinary open access archive for the deposit and dissemination of scientific research documents, whether they are published or not. The documents may come from teaching and research institutions in France or abroad, or from public or private research centers.
L'archive ouverte pluridisciplinaire HAL, est destinée au dépôt et à la diffusion de documents scientifiques de niveau recherche, publiés ou non, émanant des établissements d'enseignement et de recherche français ou étrangers, des laboratoires publics ou privés. 
M. Defoort - K.J. Lulla · C. Blanc

· H. Ftouni · O. Bourgeois · E. Collin

\section{Stressed silicon nitride nanomechanical resonators at helium temperatures}

19.06 .2012

Keywords Nanomechanical resonators, nonlinear, magnetomotive

Abstract We have characterized the mechanical resonance properties (both linear and nonlinear) of various doubly-clamped silicon nitride nanomechanical resonators, each with a different intrinsic tensile stress. The measurements were carried out at $4 \mathrm{~K}$ and the magnetomotive technique was used to drive and detect the motion of the beams. The resonant frequencies of the beams are in the megahertz range, with quality factors of the order of $10^{4}$. We also measure the dynamic range of the beams and their nonlinear (Duffing) behaviour.

PACS numbers: 74.70.Tx,74.25.Ha,75.20.Hr

\section{Introduction}

It has been known for some time now that by tuning the tensile force on a nanomechanical beam, it is possible to tune the resonant frequency and quality factor $(Q)$ of the device ${ }^{1}$. In this sense, silicon nitride is a very interesting material as it is possible to induce in it a significant amount of tensile stress by adjusting the process by which the layer is deposited. It is therefore of no surprise that in recent years, high-stress silicon nitride nanomechanical resonators have attracted considerable interest in the nanomechanics community. A number of experimental studies $^{2,3,4,5,6,7}$ have reported extremely high $Q$ factors (low mechanical damping) in these devices. The high $Q$ factors, combined with their extremely small masses and high resonant frequencies make them the ideal candidates for studying fundamental physics in the purely classical ${ }^{8,9}$ and quantum regimes ${ }^{10}$. Furthermore, their use has also been demonstrated as ultrasensitive probes for mass ${ }^{11}$ and temperature $^{12}$ sensing applications.

Institut Néel, CNRS et Université Joseph Fourier,

BP 166, 38042 Grenoble Cedex 9, France

E-mail: eddy.collin@grenoble.cnrs.fr 


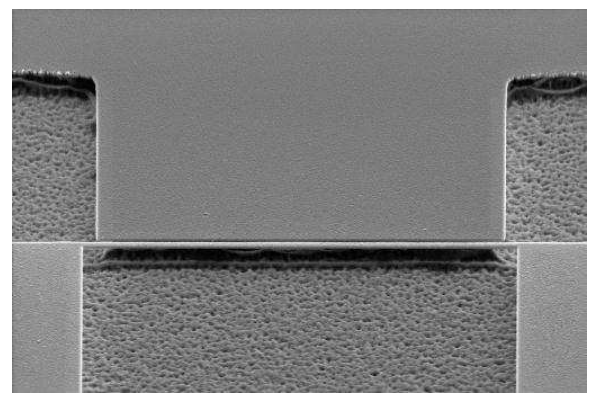

Fig. 1 (Color online) Scanning electron microscope image of a typical silicon nitride nanomechanical resonator used for the experiments described in this paper. The doubly-clamped beam has dimensions $l=15 \mu \mathrm{m}, w=250 \mathrm{~nm}, t=100 \mathrm{~nm}$, and is coated with $30 \mathrm{~nm}$ of aluminium.

In this paper, we report on measurements performed in vacuum at low temperatures on silicon nitride doubly-clamped nanomechanical resonators. We present results on two samples of similar dimensions but with different in-built tensile stresses. We characterize the mechanical properties of the beams (both in the linear and nonlinear regimes of operations) as a function of this axial load and demonstrate that these devices can be very useful for ultra low temperature experiments, much like other structures of similar dimensions previously studied ${ }^{13}$.

\section{Experimental details}

Our devices were fabricated as doubly-clamped beams on wafers composed of a silicon substrate with a $100 \mathrm{~nm}$ of LPCVD $^{1}$ silicon nitride layer on top. The wafers were first cut into $1.5 \times 1.5 \mathrm{~cm}$ pieces and, after cleaning, were coated with a 100 $\mathrm{nm}$ thick layer of PMMA (positive e-beam resist). The devices were defined using e-beam lithography and the PMMA was then developed in MIBK:IPA solution in the volume ratio 1:3 for $30 \mathrm{~s}$, followed by a quick rinse in IPA. The samples were then coated with $30 \mathrm{~nm}$ of aluminium using an e-beam evaporator, keeping the pressure below $10^{-6}$ mbar during the evaporation. The remaining resist was removed by placing the samples in N-methyl-2-pyrrolidinone solution for 1 hour at $80^{\circ} \mathrm{C}$. Finally, the beams were released via dry etching: this involved an anisotropic etch of the silicon nitride in a $\mathrm{SF}_{6}$ plasma (RIE) followed by an $\mathrm{XeF}_{2}$ etch of the underlying silicon for about 15 minutes, at a pressure of 2 Torr. The SEM image of a typical doubly-clamped beam fabricated using the technique described here is shown in Fig. 1. The pad near the beam is a gate electrode: by applying a dc voltage to it, it is possible to tune the mechanical properties of the beam ${ }^{14}$.

The devices were placed in a helium cryostat, inside a small vacuum can, and all the experiments were carried out at $4.2 \mathrm{~K}$. The resonators were part of an highimpedance electrical circuit ${ }^{15}$, their electrical resistance $r$ being around $80 \Omega$ (at $4.2 \mathrm{~K}$ using a 4-wire measurement). The motion of the beams was actuated and detected using the magnetomotive transduction scheme ${ }^{16}$. The high impedance

\footnotetext{
${ }^{1}$ Low pressure chemical vapour deposited
} 


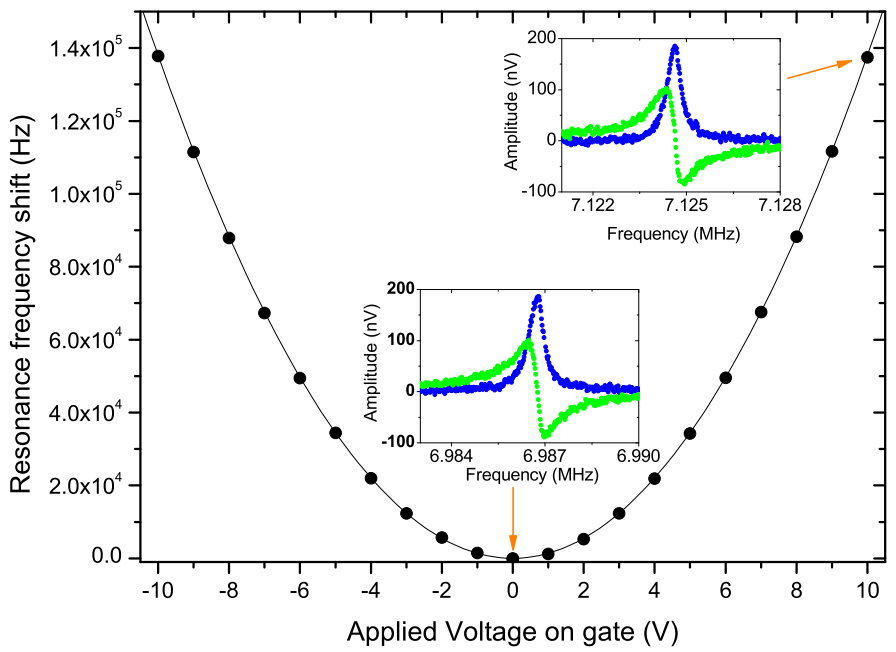

Fig. 2 (Color online) Frequency tuning of the resonance of A1 using a dc voltage on the gate electrode. For this measurement, the device motion was kept in the linear regime. No major change was observed on the $Q$ factor of the resonator. The full line is a quadratic function ${ }^{15}$. The error bars are smaller than the symbols. Inset: resonance lines (in-phase and out-of-phase components) obtained in the linear regime.

circuit means that the correction to be applied to the measured resonance parameters (frequency, linewidth) due to the magnetomotive technique ${ }^{16}$ is very small, and we can therefore extract the properties of these devices very accurately. From the resonance lineshapes, we extracted the frequencies and $Q$ factors of the beams in the linear regime. Furthermore, by changing the drive current, magnetic field and the voltage on the gate electrode, we fully characterized the linear and nonlinear properties of the devices.

\section{Results}

Two resonators were studied, one with a stress of about $100 \mathrm{MPa}$ (hereafter called sample A1) and the second one with a stress of $600 \mathrm{MPa}$ (sample A2). Device A2 had a resonant frequency of $14 \mathrm{MHz}$ and an intrinsic (unloaded) $Q$ of about 24000 , whereas A1 had a frequency of $7 \mathrm{MHz}$ and a $Q$ of 16500 . This confirms the large impact of the in-built tensile stress on the mechanical properties of such small devices and is consistent with previous studies on longer silicon nitride nano-strings ${ }^{1}$. By applying a dc voltage on the gate electrode, we were able to significantly shift the resonant frequencies of the beams, as shown with the example curve in Fig. 2 for device A1. The ability to tune the mechanical resonance of the beam with a gate electrode allows one, for instance, to make use of parametric 


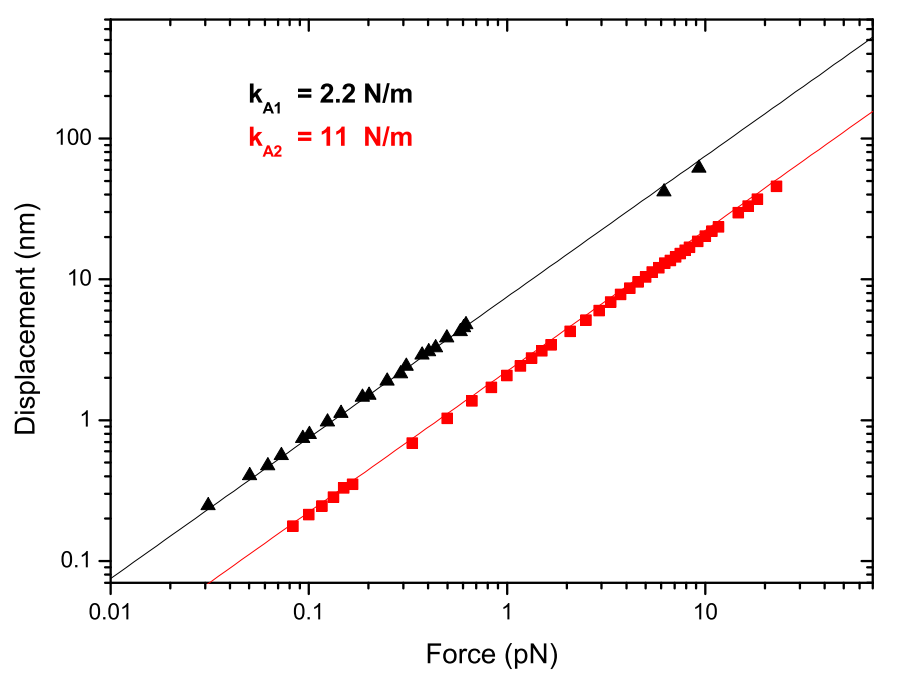

Fig. 3 (Color online) Log-log plot of the displacement versus force curves for both devices. The straight lines are fits to the experimental data from which we obtain the spring constant $k$ of the beams. The values extracted here are consistent with theory. The error bars are smaller than the symbols.

amplification ${ }^{17}$. However, no significant effect was observed in the $Q$ factor of the resonator, or even in its nonlinearity as has been observed in a previous device of similar size ${ }^{14}$. This is most likely due to the fact that the beams are already under considerable amount of stress, and the dc voltage applied to the gate (up to $10 \mathrm{~V}$ here) is not enough compared to this intrinsic stress.

Fig. 3 shows the characteristic displacement versus force data obtained for the samples measured. Both the displacement and force have been calculated in real units after careful calibrations of the signal injection and detection lines as explained in detail elsewhere ${ }^{15}$. Both devices demonstrate an extremely large dynamic range (before any plasticity appears), at least up to about $50 \mathrm{~nm}$, which is nearly half of the total thickness of the beams. The straight lines are fits to the data from which we determine the spring constant. All the values measured (frequency, spring constant and mass) are in very good agreement with the values calculated (typically $\pm 10 \%$ ).

We also characterized the beams in the nonlinear regime by driving them at large displacements. Although no change in the $Q$ factor was observed, the resonance lineshapes of the resonators became increasingly assymetric, showing hysteresis and bistability at the largest drives (inset Fig. 4). The nonlinearity observed in these devices is the typical Duffing type nonlinearity; a restoring force proportional to $x^{3}$ has to be included in the dynamics equation. This nonlinearity renormalizes the natural frequency of the resonator by a factor $\beta x^{2}$, where $\beta$ is 


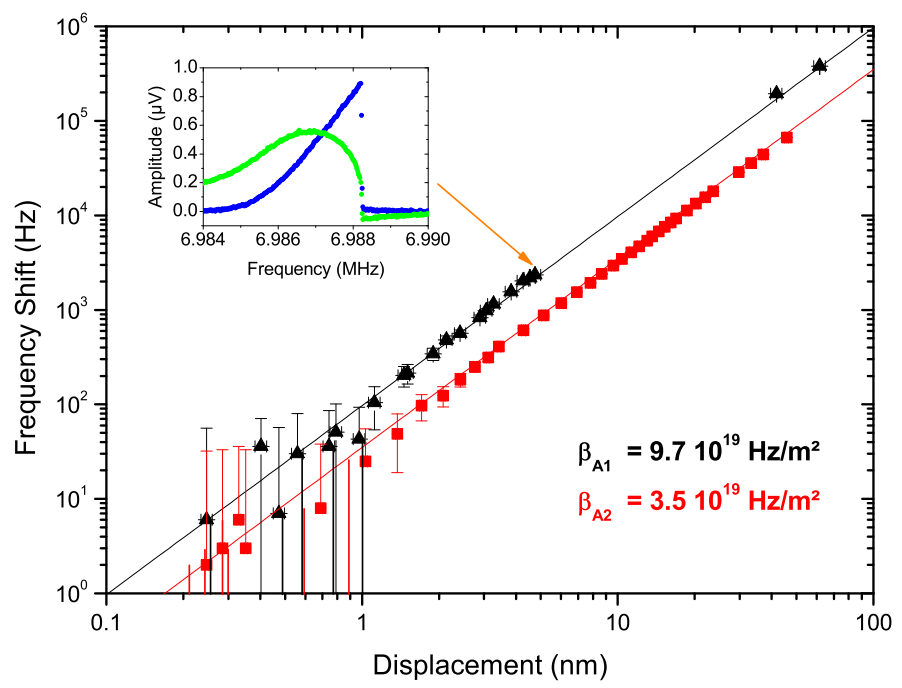

Fig. 4 (Color online) Log-log plot of the frequency versus displacement for both devices. The full lines are quadratic fits to the data from which we determine the nonlinear (Duffing) term, $\beta$, which appears for large displacements. Inset: example resonance line in the nonlinear regime.

the nonlinear coefficient, and can be positive or negative depending on whether the frequency shifts up or down ${ }^{18}$. As seen in Fig. 4, the frequency of our devices shifts upwards, demonstrating a positive nonlinearity. From the quadratic fits to the data, we determine the nonlinear coefficients, which are in very good agreement (within 10\%) with theory ${ }^{19}$.

\section{Conclusions}

We have measured the mechanical properties of two silicon nitride nanomechani$\mathrm{cal}$ resonators at $4 \mathrm{~K}$. The doubly-clamped beams were fabricated using a combination of e-beam lithography and dry etching techniques. Their motion was actuated and detected using the magnetomotive technique. The two resonators vibrate at megahertz frequencies with $Q$ factors of the order of $10^{4}$, the one with the larger stress having a larger frequency and $Q$, which is consistent with previous studies on silicon nitride structures. Furthermore, both beams exhibit a very large dynamic range, of at least $50 \mathrm{~nm}$ which is quite remarkable taking into account they are only about $100 \mathrm{~nm}$ thick. At large displacements, the beams enter the nonlinear regime with the resonance lineshapes becoming increasingly assymetric, as well as bistable. In this regime, the nonlinearity comes from the displacement induced stress in the beams, which adds to the intrinsic stress already present in them. This 
nonlinear behaviour is well described by adding a Duffing type nonlinearity to the equation of motion of the beams.

Finally, through our measurements we have fully characterized our devices, and obtained their resonant frequencies, $Q$ factors, spring constants and nonlinear coefficients in real units. This is extremely useful for future experiments with such devices, at even lower temperatures where quantum effects might start to play a key role.

Acknowledgements We would like to thank G. Julie, E. Andre and T. Fournier for help with the fabrication of the devices. We acknowledge the support from MICROKELVIN, the EU FRP7 low temperature infrastructure grant 228464, and of the 2010 ANR French grant QNM n ${ }^{\circ} 0404$ 01 .

\section{References}

1. S.S. Verbridge, D.F. Shapiro, H.G. Craighead, and J.M. Parpia, Nano Lett. 7, 1728 (2007).

2. S.S. Verbridge, J.M. Parpia, R.B. Reinchenbach, L.M. Bellan, and H.G. Craighead, J. Appl. Phys. 99, 124304 (2006).

3. S.S. Verbridge, H. G. Craighead, and J.M. Parpia, Appl. Phys. Lett. 92, 013112 (2008).

4. D.R. Southworth, R.A. Barton, S.S. Verbridge, B. Ilic, A.D. Fefferman, H.G. Craighead, and J.M. Parpia, Phys. Rev. Lett. 102, 225503 (2009).

5. Q.P. Unterreithmeier, T. Faust, and J.P. Kotthaus, Phys. Rev. Lett. 105, 027205 (2010).

6. S. Schmid, K.D. Jensen, K. H. Nielsen, and A. Boisen, Phys. Rev. B 84, 165307 (2011)

7. A. Suhel, B.D. Hauer, T.S. Biswas, K.S.D. Beach, and J.P. Davis, Appl. Phys. Lett. 100, 173111 (2012).

8. Q.P. Unterreithmeier, E.M. Weig, and J.P. Kotthaus, Nature 458, 1001 (2009).

9. K.Y. Fong, W.H.P. Pernice, and H.X. Tang, Phys. Rev. B 85, 161410 (2012).

10. T. Rocheleau, T. Nkudum, C. Macklin, J.B. Hertzberg, A.A. Clerk, and K.C. Schwab, Nature 463, 72 (2010).

11. R.A. Barton, B. Ilic, S.S. Verbridge, B.R. Cipriany, J.M. Parpia, and H. G. Craighead, Nano Lett. 10, 2058 (2010).

12. T. Larsen, S. Schmid, L. Grönberg, A.O. Niskanen, J. Hassel, S. Dohn, A. Boisen, Appl. Phys. Lett. 98, 121901 (2011).

13. E. Collin, T. Moutonet, J.-S. Heron, O. Bourgeois, Yu.M. Bunkov, and H. Godfrin, J. Low Temp. Phys. 162, 653 (2011).

14. I. Kozinsky, H.W.Ch. Postma, I. Bargatin, and M.L. Roukes, Appl. Phys. Lett. 88, 253101 (2006).

15. E. Collin, M. Defoort, K. Lulla, T. Moutonet, J.-S. Heron, O. Bourgeois, Yu.M. Bunkov, and H. Godfrin, Rev. Sci. Instrum. 83, 045005 (2012).

16. A.N. Cleland, and M.L. Roukes, Sensors and Actuators 72, 256 (1999).

17. E. Collin, T. Moutonet, J.-S. Heron, O. Bourgeois, Yu.M. Bunkov, and H. Godfrin, Phys. Rev. B 84, 054108 (2011).

18. E. Collin, Yu.M. Bunkov, and H. Godfrin, Phys. Rev. B 82, 235416 (2010). 
19. A.H. Nayfeh and D.T. Mook, Nonlinear Oscillations, Wiley Classics Library, VCH (1995). 